University of New Orleans

ScholarWorks@UNO

\title{
Application of plasma resonance condition for prediction of large Kerr effects
}

A. De

University of New Orleans

A. Puri

University of New Orleans

Follow this and additional works at: https://scholarworks.uno.edu/phys_facpubs

Part of the Physics Commons

\section{Recommended Citation}

J. Appl. Phys. 92, 5401 (2002)

This Article is brought to you for free and open access by the Department of Physics at ScholarWorks@UNO. It has been accepted for inclusion in Physics Faculty Publications by an authorized administrator of ScholarWorks@UNO.

For more information, please contact scholarworks@uno.edu. 


\title{
Application of plasma resonance condition for prediction of large Kerr effects
}

\author{
A. De and A. Puria \\ Department of Physics, University of New Orleans, New Orleans, Louisiana 70148
}

(Received 19 April 2002; accepted for publication 24 July 2002)

\begin{abstract}
Resonance like enhancement of magneto-optic Kerr effects (MOKEs) have been attributed to numerous effects, one of them being plasma resonance of free charge carriers, which is analytically and numerically investigated here. Analytical expressions for frequency dependent enhancement of MOKE are obtained in the general framework of the Drude model, which are then applied to well known expressions describing MOKE. The derived expressions are numerically tested for various optical constants. It is known that for certain materials, the resonance like enhancements in the Kerr spectrum occur in the near vicinity of $\operatorname{Re}\left[\varepsilon_{x x}\right]=1$, which is generally near the plasma edge. This is seen to be true when the plasma frequency $\omega_{p}$ is greater in magnitude as compared to the cyclotron frequency $\omega_{c}$, i.e., if $\omega_{p} \gg \omega_{c}$, whereas in the event of a large reflection edge split, i.e., if $\omega_{p}$ $\sim \omega_{c}$ the resonance like peaks will occur near $\operatorname{Re}\left[\varepsilon_{+} \cdot \varepsilon_{-}\right] \approx 1$, which is a relatively more general condition as compared to $\operatorname{Re}\left[\varepsilon_{x x}\right]=1$. Second, we see through model calculations that the spectral proximity of the MOKE resonance peak to the plasma edge is also subject to the magnitude of the background dielectric constant. Results are explained analytically and numerically. Good agreement is obtained between the expressions derived here and the numerically observed occurrence of resonance like peaks in the Kerr spectrum. (c) 2002 American Institute of Physics.
\end{abstract}

[DOI: 10.1063/1.1507816]

\section{INTRODUCTION}

In the last half of the 20th century, a vast amount of research has been carried out in the field of magneto-optics. Numerous experiments have been devised to study the band structure of various semiconductor materials making use of various magneto-optical effects such as magneto-plasma reflection, rotation, ellipticity, cyclotron resonance, Faraday, Kerr, and Voigt effects. Of particular interest, is the magnetooptic Kerr effect (MOKE), particularly the polar Kerr effect. Its importance pertains mainly to the optical readout of magnetically stored information and to other technological applications.

It is worth noting that the classical model proposed by Drude, ${ }^{1}$ nearly $100 \mathrm{yr}$ ago, has been carried over essentially intact and can still be used to quantitatively explain much of today's MOKE experiments. Excellent agreement between the calculated spectra, based on the Drude model, and experimental data has been seen for $n$-type $\mathrm{InSb},{ }^{2}$ paramagnetic silver, ${ }^{3}$ and for $\mathrm{Y}-\mathrm{Ba}-\mathrm{Cu}-\mathrm{O}$ crystals. ${ }^{4}$ Calculated Kerr rotation (KR) spectra of $\mathrm{NdS}^{5}$ and thulium monochalcogenides $(\mathrm{TmS} \text { and } \mathrm{TmSe})^{6}$ were found to be in good agreement, by taking the cyclotron frequency $\omega_{c}$ as a free parameter to fit the experimental data. Explanations on the origin of large band-splitting effects seen at $3.3 \mathrm{eV}$ for $\mathrm{Bi}$ substituted yttrium iron garnet (YIG) samples ${ }^{7}$ have been provided based on the Drude model. There are of course fine structure and new effects of an oscillatory nature, which can only be explained on the basis of quantum theory. For a qualitative

\footnotetext{
a) Author to whom correspondence should be addressed; electronic mail: apuri@uno.edu
}

comparison of different methods used for calculating the magneto-optic spectra one can refer to Delin et al. ${ }^{8}$

The appearance and prediction of high KR peaks in the MOKE spectrum has generated much interest. This has been attributed to various physical origins such as interband transitions and spin orbit coupling. ${ }^{9,10} \mathrm{KR}$ enhancement in the case of ferromagnetic layers embedded in metallic matrices has been more widely attributed to plasma resonance of free carriers in the nonmagnetic noble metals ${ }^{11,12}$ and also to sharp surface plasmon resonance of noble metals in total reflection geometry. ${ }^{13}$ In the case of a single substrate, Feil and Hass $(\mathrm{FH})^{14}$ have suggested a different explanation for large MOKE. They have shown through model calculations that large MOKE occur wherever $\operatorname{Re}\left[\varepsilon_{x x}\right] \approx 1$, which according to them is in the vicinity of plasma resonance frequency of free charge carriers. FH's condition for MOKE resonance was, however, disputed by Schones and Reim. ${ }^{15,16}$ Objections were raised on issues such as the validity of assuming $\varepsilon_{x y}$ to be a constant, which was said to be inconsistent with Kramer-Kronig relations.

The purpose of this article is threefold. First, we wish to address some of the issues in Refs. 15 and 16, specifically, the role of the damping constant in $\operatorname{Im}\left[\varepsilon_{x x}\right]$. This is carried out in the framework of Drude model-based dielectric tensor elements, to be self-consistent with Kramer-Kronig relations. It is also shown numerically and analytically that the spectral proximity of the MOKE resonance peak to the plasma edge is subject to the magnitude of the background dielectric constant (especially at lower values).

Second in view of the above-mentioned points, we obtain an expression using the classical Drude model and the FH condition which enables us to predict the optical fre- 
quency at which resonance like enhancements in the Kerr spectrum occur. This analytical expression is then compared against numerically obtained spectral locations of the MOKE peaks over a range of optical constants, to cover various possibilities. This comparison is also extended to the $\varepsilon_{ \pm}$ $=1$ condition, which corresponds to the reflectivity minima of right/left circularly polarized light and is in close spectral vicinity of the MOKE maxima.

Finally, in the process of comparative numerical analysis, it is shown that the $\operatorname{Re}\left[\varepsilon_{x x}\right] \approx 1$ condition is inaccurate in predicting the spectral occurrence of the MOKE peaks in the event of a large reflection edge split. An immediate implication of this is that the much used MOKE expression, which is instrumental to the FH condition, has its own shortcomings when $\omega_{c} \sim \omega_{p}$. Alternatively, it is shown that in the event of a large reflection edge split, a derived expression based on the $\operatorname{Re}\left[\varepsilon_{+} \varepsilon_{-}\right]=1$ condition is more effective in predicting the spectral occurrence of MOKE peaks. Analytical explanations are provided, wherever necessary, for a better understanding of the numerical results.

\section{MAGNETO-OPTICAL KERR EFFECT AND THE DRUDE MODEL}

It is well known that dielectric response of the medium can be described by Maxwell's equations. The propagation of an electromagnetic wave in a material can be described by the electric and magnetic permeability tensors $\varepsilon$ and $\mu$. By ignoring nonlinear effects in the material response and assuming that $\mu=1$ at optical frequencies, the complex dielectric tensor can be simplified (due to symmetry of the problem configuration) and expressed as

$$
\varepsilon=\begin{array}{ccc}
\varepsilon_{x x} & i \varepsilon_{x y} & 0 \\
-i \varepsilon_{x y} & \varepsilon_{y y} & 0 \\
0 & 0 & \varepsilon_{z z}
\end{array} .
$$

This leads to the following expression for the complex index of refraction:

$$
\tilde{n}_{ \pm}^{2}=\varepsilon_{x x} \pm \mathrm{i} \varepsilon_{x y}=\varepsilon_{ \pm},
$$

where, the + and - signs, respectively, represent right and left circularly polarized modes of propagation of an electromagnetic wave in a longitudinal magnetic field, $\tilde{n}_{ \pm}$is the complex index of refraction given by $\tilde{n}_{ \pm}=n_{ \pm}-i k_{ \pm}, n_{ \pm}$and $k_{ \pm}$are the refractive and absorptive parts, respectively.

In the polar Kerr configuration, the magnetization is perpendicular to the surface and parallel to the direction of light propagation. The eigenmodes are left and right circular polarizations and therefore the KR can be expressed as the phase difference between left and right circularly polarized light $^{17}$

$$
\theta_{K}=-\frac{1}{2}\left(\Delta_{+}-\Delta_{-}\right)
$$

$\mathrm{KE}$ is expressed in terms of the complex reflection coefficients as

$$
\xi_{K}=-\frac{\left|r_{+}\right|-\left|r_{-}\right|}{\left|r_{+}\right|+\left|r_{-}\right|} .
$$

The phase difference for left and right circularly polarized light is given by

$$
\Delta_{ \pm}=\tan ^{-1}\left(\frac{-2 k_{ \pm}}{n_{ \pm}^{2}+k_{ \pm}^{2}-1}\right)=-j \log \frac{r_{ \pm}}{\left|r_{ \pm}\right|},
$$

where the Fresnel's reflection coefficient at normal incidence is given by

$$
r_{ \pm}=\frac{1-\tilde{n}_{ \pm}}{1+\tilde{n}_{ \pm}} .
$$

Assuming that $\left(\Delta_{+}-\Delta_{-}\right)$is small and $\left(r_{+}-r_{-}\right)^{2}$ $\ll 2 r_{+} \cdot r_{-}$, expressions for the complex polar MOKE given by Eqs. (3) and (4) can be approximated to ${ }^{18,19}$

$$
\Theta_{k}=\theta_{k}-i \xi_{k}=i \frac{\tilde{n}_{+}-\tilde{n}_{-}}{\tilde{n}_{+} \tilde{n}_{-}-1} .
$$

By using the properties of the material's dielectric tensor and further assuming that $\varepsilon_{x x} \gg \varepsilon_{x y},{ }^{18}$ one obtains the widely used expression for the MOKE

$$
\Theta_{k}=\theta_{k}-i \xi_{k}=\frac{i \varepsilon_{x y}}{\left(1-\varepsilon_{x x}\right) \sqrt{\varepsilon_{x x}}} .
$$

The Drude model is used here to describe the diagonal and off diagonal components of the dielectric tensor. In the Drude model, the application of a magnetic field splits the plasma reflection edge into two. The spectral separation between the two reflectivity minima increases linearly ${ }^{20}$ with increasing magnetic field, thus changing the complex index of refraction and inducing magnetic circular bifringence, and magnetic circular dichroism. In the Kerr configuration with the Poynting vector $S \| B_{0}$ and $E \perp B_{0}$, the complex dielectric tensor is expressed as ${ }^{1}$

$$
\varepsilon_{ \pm}=\varepsilon_{\infty}\left(1-\frac{\omega_{p}^{2}}{\omega\left(\omega \pm \omega_{c}-i \gamma\right)}\right),
$$

where, $\omega_{p}=\left(4 \pi N e^{2} / m^{*} \varepsilon_{c}\right)^{1 / 2}$ is the plasma frequency, $\omega_{c}$ $=\mathrm{He} / \mathrm{m}^{*} \mathrm{c}$ is the cyclotron frequency, $\gamma$ is the scattering frequency (or damping constant), and $\varepsilon_{\infty}$ is the background dielectric constant, $\mathrm{H}$ being the magnetic field, $\mathrm{c}$ the speed of light, e the charge of an electron, $m^{*}$ the effective mass, $\mathrm{N}$ the doping concentration, and $\varepsilon_{c}$ the dielectric constant of the medium.

The diagonal and off-diagonal components of the dielectric tensor can be obtained from Eqs. (2) and (9) and are expressed in terms of their respective real and imaginary parts as

$$
\begin{aligned}
\varepsilon_{x x}= & \varepsilon_{\infty}\left(1-\frac{\omega_{p}^{2}\left(\omega^{2}-\omega_{c}^{2}+\gamma^{2}\right)}{\left(\omega^{2}-\omega_{c}^{2}-\gamma^{2}\right)^{2}+4 \gamma^{2} \omega^{2}}\right. \\
& \left.+i \frac{\gamma \omega_{p}^{2}\left(\omega^{2}+\omega_{c}^{2}+\gamma^{2}\right)}{\left[\left(\omega^{2}-\omega_{c}^{2}-\gamma^{2}\right)^{2}+4 \gamma^{2} \omega^{2}\right] \omega}\right)
\end{aligned}
$$




$$
\begin{aligned}
\varepsilon_{x y}= & \varepsilon_{\infty}\left(\frac{-2 \gamma \omega_{c} \omega_{p}^{2}}{\left(\omega^{2}-\omega_{c}^{2}-\gamma^{2}\right)^{2}+4 \gamma^{2} \omega^{2}}\right. \\
& \left.+i \frac{\omega_{c} \omega_{p}^{2}\left(\omega^{2}-\omega_{c}^{2}-\gamma^{2}\right)}{\left[\left(\omega^{2}-\omega_{c}^{2}-\gamma^{2}\right)^{2}+4 \gamma^{2} \omega^{2}\right] \omega}\right) .
\end{aligned}
$$

It is apparent from Eq. (8) and as pointed out by $\mathrm{FH}^{14}$ that for a non-negligible $\varepsilon_{x y}$, a large resonance like enhancement of MOKE is expected in the frequency region where $\operatorname{Re}\left[\varepsilon_{x x}\right] \approx 1$ and under the assumption that $\operatorname{Im}\left[\varepsilon_{x x}\right] \approx 0$. We illustrate this point by using the simple Drude model and closely examining $\operatorname{Im}\left[\varepsilon_{x x}\right]$ from Eq. (10). By inspection, it can be deduced that in order for $\operatorname{Im}\left[\varepsilon_{x x}\right] \approx 0$, the damping constant $\gamma$ needs to be small. In the numerical example in Ref. $15, \gamma$ was chosen to be $1 \mathrm{eV}$ (as pointed out by $\mathrm{FH},{ }^{16}$ which invalidates the assumption that $\operatorname{Im}\left[\varepsilon_{x x}\right] \approx 0$, and therefore no MOKE enhancement was observed in the vicinity of $\operatorname{Re}\left[\varepsilon_{x x}\right]=1$.

Presently, we are interested in obtaining an expression for the optical frequency at which large resonance like enhancements in the Kerr spectrum occur. This is initially done by using the FH condition and obtaining an expression by equating $\operatorname{Re}\left[\varepsilon_{x x}\right]=1$ in Eq. (10). By solving for the roots of $\omega$, we obtain

$$
\omega=\left[\frac{\omega_{p}^{2}}{2 x}+\left(\omega_{c}^{2}-\gamma^{2}\right) \pm\left(\frac{\omega_{p}^{4}}{4 x^{2}}-4 \gamma^{2} \omega_{c}^{2}\right)^{1 / 2}\right]^{1 / 2},
$$

where $x=1-1 / \varepsilon_{\infty}$.

By ignoring $\gamma^{2}$ terms in Eq. (12) and choosing the root with the + sign, we simplify the above expression and denote by $\omega_{o 1}$,

$$
\omega_{o 1}=\sqrt{\frac{\omega_{p}^{2}}{x}+\omega_{c}^{2}} .
$$

The following expression where the $\gamma^{2}$ terms have not been ignored will also be used in our comparative study for the sake of completeness. We denote this as $\omega_{o 2}$ and choose the root with the + sign,

$$
\omega_{o 2}=\left[\frac{\omega_{p}^{2}}{2 x}+\left(\omega_{c}^{2}-\gamma^{2}\right)+\left(\frac{\omega_{p}^{4}}{4 x^{2}}-4 \gamma^{2} \omega_{c}^{2}\right)^{1 / 2}\right]^{1 / 2} .
$$

It is interesting to note that by choosing the root with the sign in Eq. (12) and assuming that $4 x \omega_{c} \cdot \gamma \ll \omega_{p}^{2}$, we obtain

$$
\omega=\left[\omega_{c}^{2}+\gamma^{2}\left(\frac{4 x \omega_{c}^{2}}{\omega_{p}^{2}}-1\right)\right]^{1 / 2} \approx \omega_{c} .
$$

Finally, it is worth noting that the spectral location of the reflectivity minima is always in close proximity of the MOKE maxima, hence an expression for $\omega$, where zero reflectivity occurs, is of interest for a comparative numerical analysis. This can be obtained by equating $\tilde{n}_{ \pm}=1$ (or $\varepsilon_{ \pm}$ $=1$ ) in Eq. $(9)^{21,22}$ and is denoted here by $\omega_{\text {or }}$

$$
\omega_{o r}=\frac{1}{2}\left[ \pm \omega_{c}-\gamma \pm\left(\frac{4 \omega_{p}^{2}}{x}+\left(\omega_{c}-i \gamma\right)^{2}\right)^{1 / 2}\right] .
$$

Equations (13), (14) and (16) will be used in the numerical analysis and comparison to follow in Sec. III.

\section{NUMERICAL RESULTS AND ANALYSIS}

It is instructive to calculate and plot the MOKE spectrum over a wide range of optical constants and parameters. In this section we carry out a comparative study between the expressions used for describing MOKE and its actual numerical spectral occurrence. Conditions responsible for the resonance like enhancements and their limitations under the framework of the Drude model are subsequently discussed. The real and imaginary parts of the diagonal component of the dielectric tensor were obtained from Eq. (10) and the off-diagonal component was obtained from Eq. (11) in order to maintain self-consistency. Wavelength dependent refractive indices, computed using Eq. (2), were used to compute the MOKE spectra using exact [Eqs. (3) and (4)] and approximate expressions [Eq. (8)].

In Fig. 1 we have shown the calculated KR, KE, reflectivity, and dielectric tensor elements as a function of incident photon energy. For illustrative purposes we choose the following optical constants: $\varepsilon_{\infty}=10, \gamma=0.1 \mathrm{eV}, \omega_{c}=0.2 \mathrm{eV}$, and $\omega_{p}=2 \mathrm{eV}$. Figure 1(a) shows the computed MOKE spectrum, using Eqs. (3) and (4). In Fig. 1(b), we plot the MOKE spectrum using Eq. (7). It can be clearly seen that the MOKE line shapes of Figs. 1(a) and 1(b) are similar, except for that in Fig. 1(b) a marginally larger MOKE enhancement is seen and that the KR peak occurs at $\omega_{o}^{\prime}=2.64 \mathrm{eV}$, whereas in the case of Fig. 1(a) it occurs at $\omega_{o}=2.66 \mathrm{eV}$. For lower values of $\omega_{c}$ the line shapes of Figs. 1(a) and 1(b) were identical. Notice in Fig. 1(f) that at $2.64 \mathrm{eV}, \operatorname{Re}\left[\varepsilon_{x x}\right]=1$, and $\operatorname{Im}\left[\varepsilon_{x x}\right]$ is small but not negligible. Nevertheless, as predicted by $\mathrm{FH}$ the $\mathrm{KR}$ is indeed optimum wherever $\operatorname{Re}\left[\varepsilon_{x x}\right]$ $\approx 1$.

Next, the above-mentioned condition is tested over a range of optical constants using Eqs. (3) and (8). We find it useful to discuss the case using Eq. (8) first. The optical frequency at which the maximum KR occurs using Eq. (8) is numerically obtained and is denoted here by $\omega_{o}^{\prime}$. This is then normalized to $\omega_{o}^{\prime} / \omega_{c}$ and plotted as a function of $\varepsilon_{\infty}$, for fixed normalized plasma frequency $\left(\omega_{p} / \omega_{c}\right)$, as shown in Fig. 2. In order to illustrate general trends, this is done for $\omega_{p} / \omega_{c}=1,2$, and 10 , respectively. The purpose here is to compare the occurrence of $\omega_{o}^{\prime} / \omega_{c}$, as obtained by using Eq. (8), against the results predicted by Eqs. (13), (14), and (16) (which are again normalized to $\omega_{o 1} / \omega_{c}, \omega_{o 2} / \omega_{c}$ and $\omega_{o r} / \omega_{c}$, respectively). From Fig. 2 it is seen that near perfect agreement is obtained between $\omega_{o 1} / \omega_{c}, \omega_{o 2} / \omega_{c}$ and $\omega_{o}^{\prime} / \omega_{c}$ with increasing $\omega_{p} / \omega_{c}$. Notice the relatively larger error for $\omega_{p} / \omega_{c}=1$ in Fig. 2(a) and that the $\omega_{o 1} / \omega_{c}$ curve is closer to the $\omega_{o}^{\prime} / \omega_{c}$ curve as opposed to the $\omega_{o 2} / \omega_{c}$ curve. This can be attributed to the fact that we use $\gamma=0.1 \mathrm{eV}$, which assumes greater significance with decreasing $\omega_{p} / \omega_{c}$. The reason for this becomes clearer when we rewrite Eq. (12) as 
(a.)

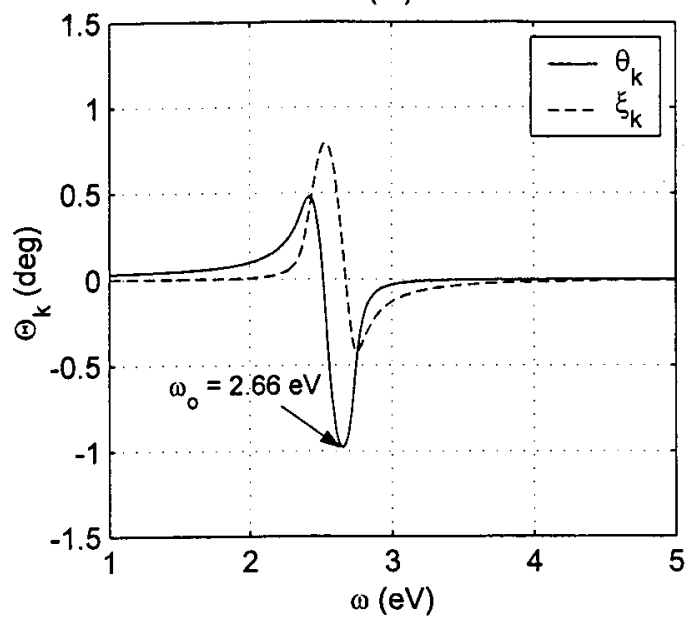

(c.)

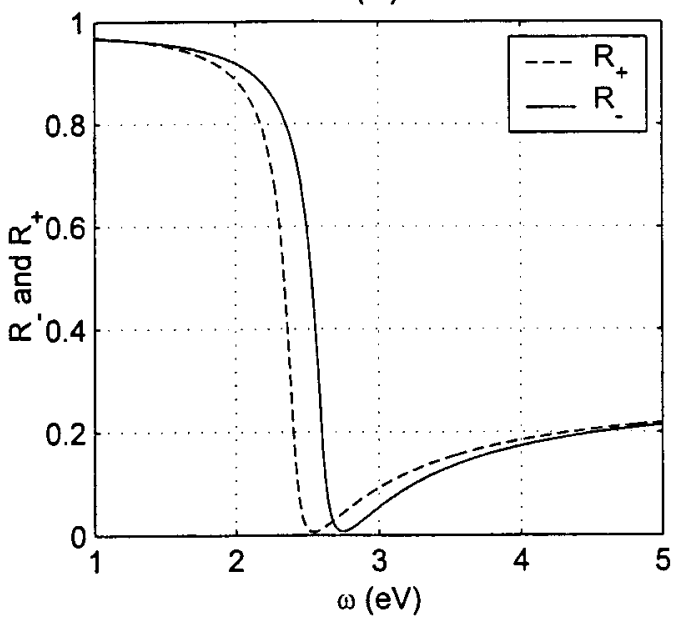

(e.)

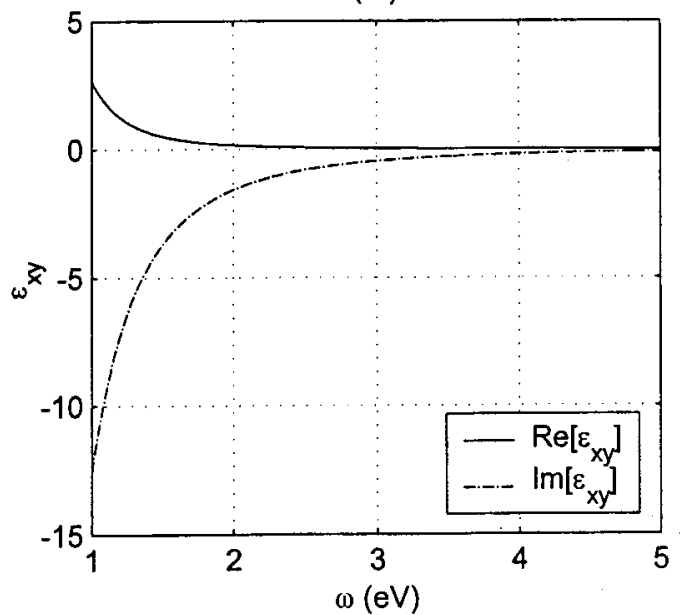

(b.)

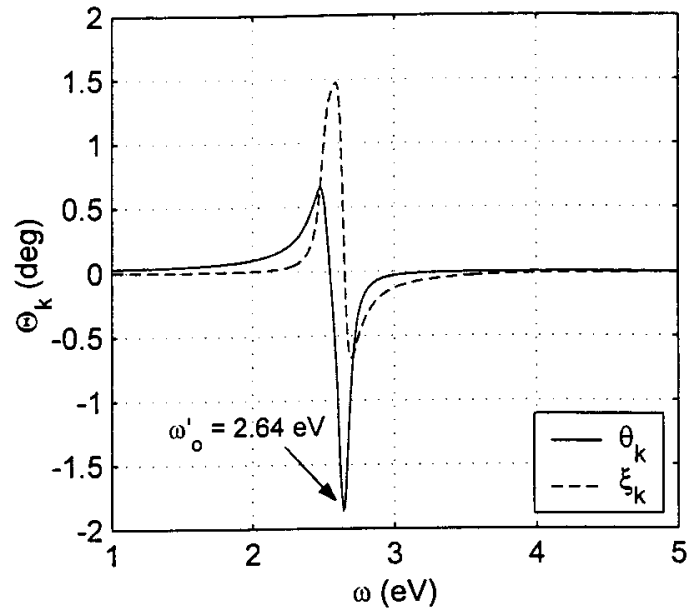

(d.)

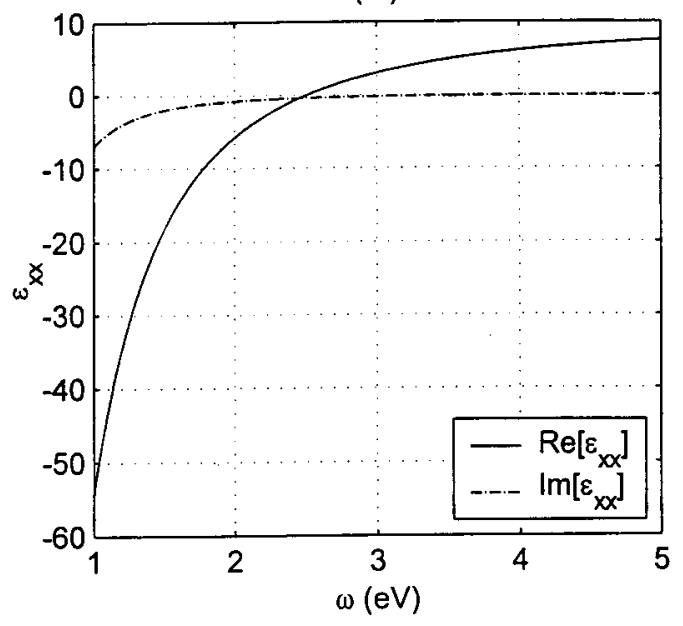

(f.)

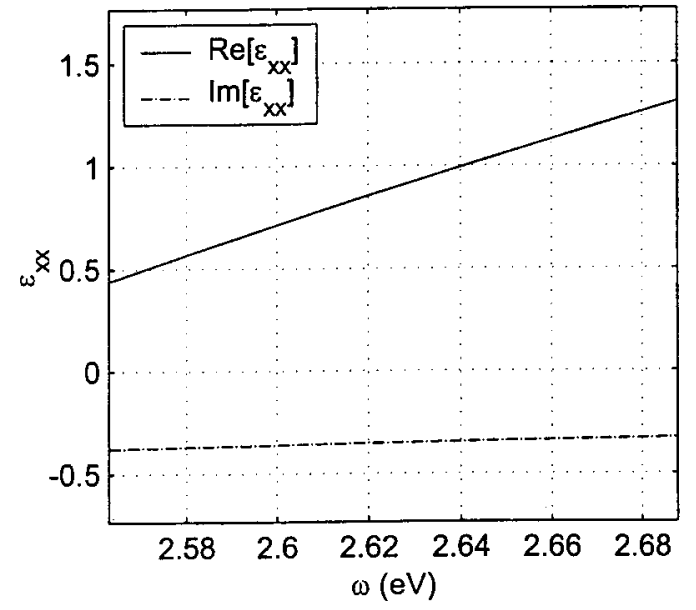

FIG. 1. (a) MOKE parameters using Eqs. (3) and (4). (b) MOKE parameters using Eq. (8). (c) Reflectivity. (d) Diagonal dielectric tensor component ( $\varepsilon_{x x}$ ). (e) Off-diagonal dielectric tensor component $\left(\varepsilon_{x y}\right)$. (f) Zoom in view of $\varepsilon_{x x}$; constants used: $\varepsilon_{\infty}=10, \omega_{p}=2.5 \mathrm{eV}, \omega_{c}=0.2 \mathrm{eV}$, and $\gamma=0.1 \mathrm{eV}$.

$$
\omega \approx\left[\frac{\omega_{p}^{2}}{x}+\omega_{c}^{2}-\gamma^{2}\left(1+\frac{4 x \omega_{c}^{2}}{\omega_{p}^{2}}\right)\right]^{1 / 2} .
$$

Our objective here is to express the coefficient of $\gamma$ as a function of $\omega_{p} / \omega_{c}$. Obviously, the damping constant $\gamma$ and its coefficient will assume a more significant role as $\omega_{p} / \omega_{c}$ decreases and also with increasing $\varepsilon_{\infty}$ (which implies that $\mathrm{x}$ $\sim 1$ ). This effect is clearly seen in Fig. 2(a) where the separation between various line shapes increases with increasing $\varepsilon_{\infty}$.

Also notice in Fig. 2, that as we approach lower $\varepsilon_{\infty}$ values, the spectral location of the KR maxima moves away 
(a.)

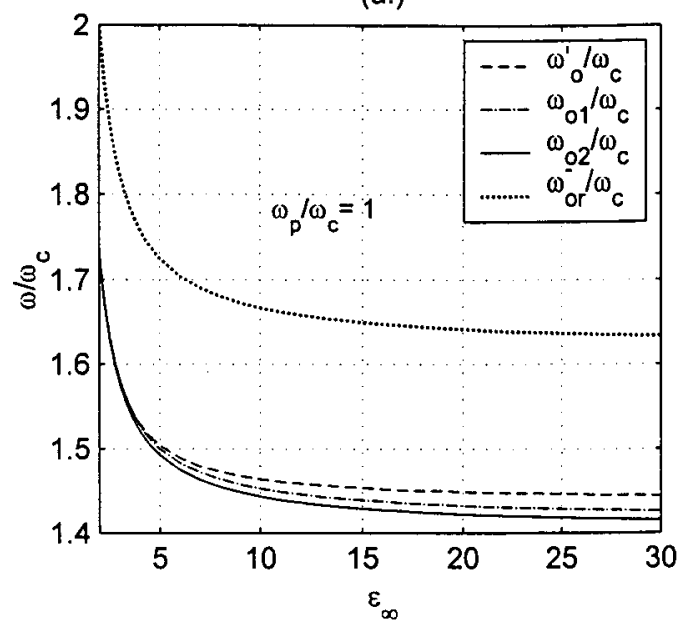

(b.)

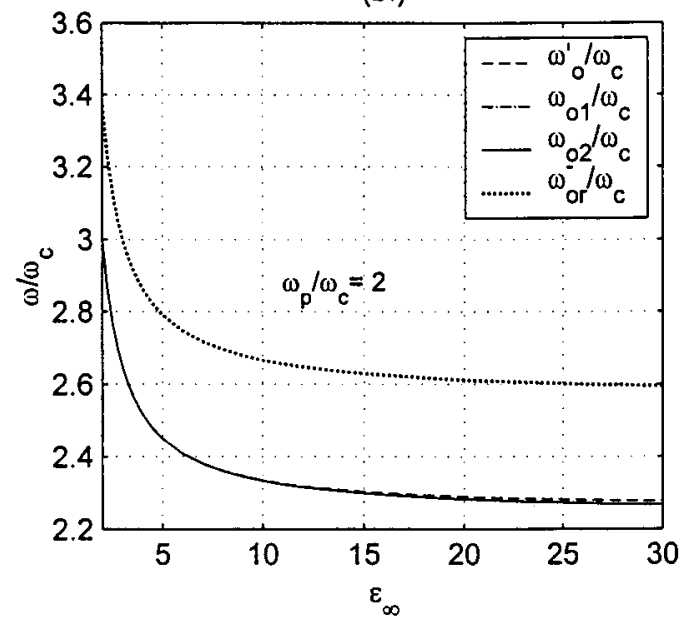

(c.)

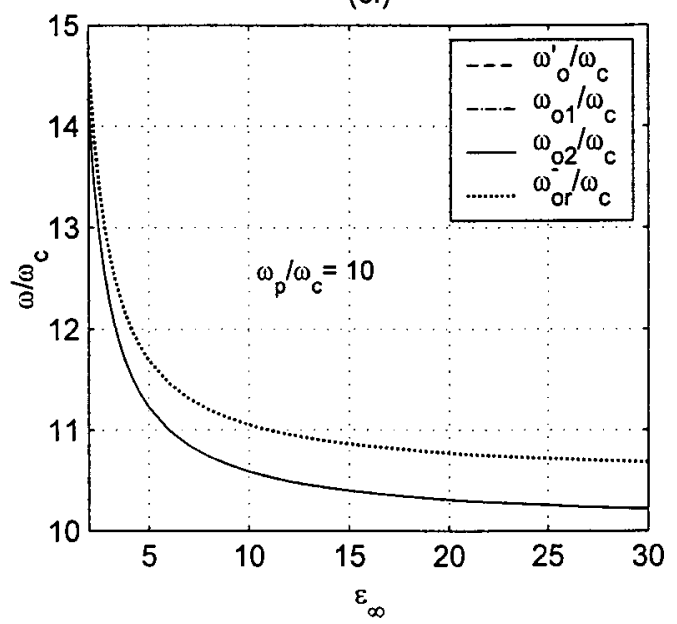

FIG. 2. Normalized comparison of the numerically obtained optical frequencies of KR maxima using Eq. (3), against Eqs. (13), (14), and (16), as a function of $\varepsilon_{\infty}$ for various $\omega_{p} / \omega_{c}$.

exponentially from the vicinity of the plasma edge. We attribute this largely to the $1 / \mathrm{x}$-dependent coefficient of $\omega_{p}^{2}$ (first term) on the right-hand side of Eq. (17). This phenomenon is seen for all numerical cases to follow.

A similar comparative study is carried out next for the numerically obtained normalized spectral location of the KR (a.)

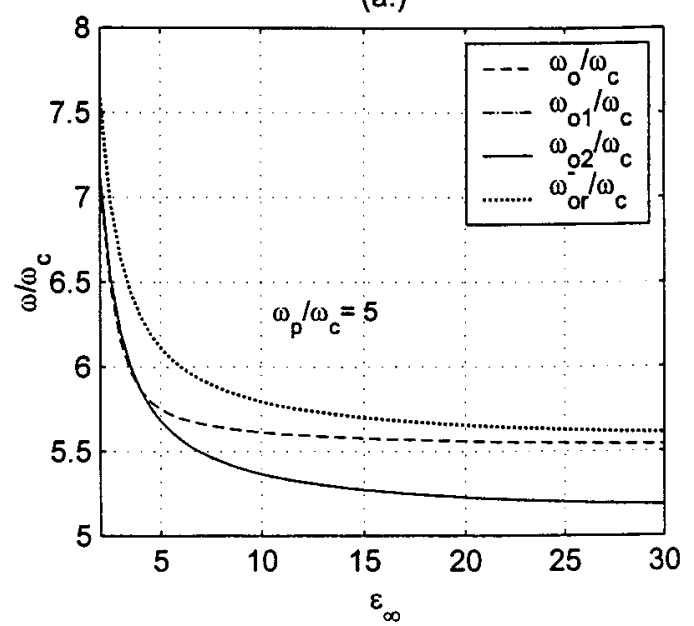

(b.)

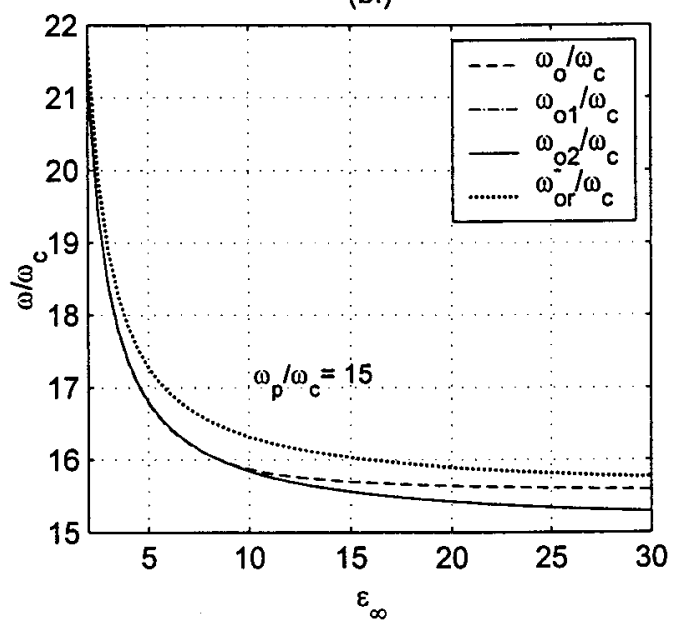

(c.)

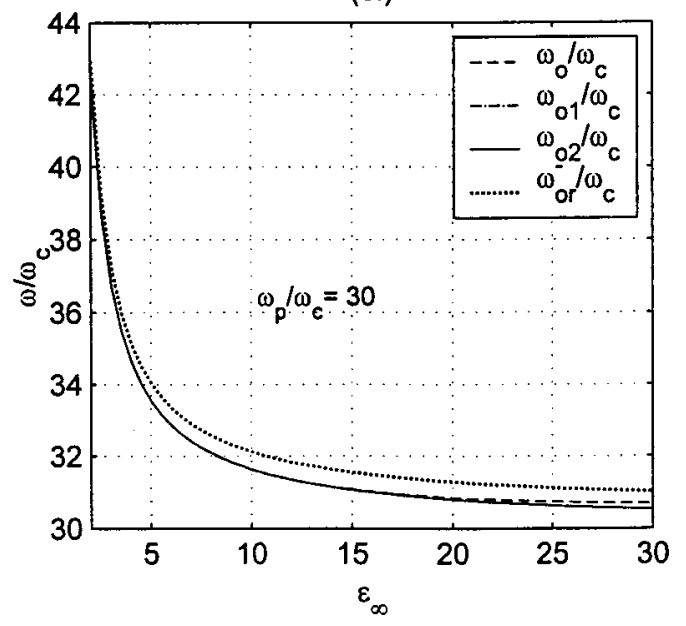

FIG. 3. Normalized comparison of the numerically obtained optical frequencies of KR maxima using Eq. (8), against Eqs. (13), (14), and (16), as a function of $\varepsilon_{\infty}$ for various $\omega_{p} / \omega_{c}$.

peaks using Eq. (3), i.e., $\omega_{o} / \omega_{c}$, and $\omega_{o 1} / \omega_{c}, \omega_{o 2} / \omega_{c}$, $\omega_{o r} / \omega_{c}$. These quantities are plotted in Fig. 3 as a function of $\varepsilon_{\infty}$ for three illustrative cases of $\omega_{p} / \omega_{c}=5,15$, and 30 , respectively. It is seen that the agreement between $\omega_{o} / \omega_{c}$, and $\omega_{o 1} / \omega_{c}, \omega_{o 2} / \omega_{c}$ is again better for higher $\omega_{p} / \omega_{c}$, however the difference between the numerically obtained 
(a.)

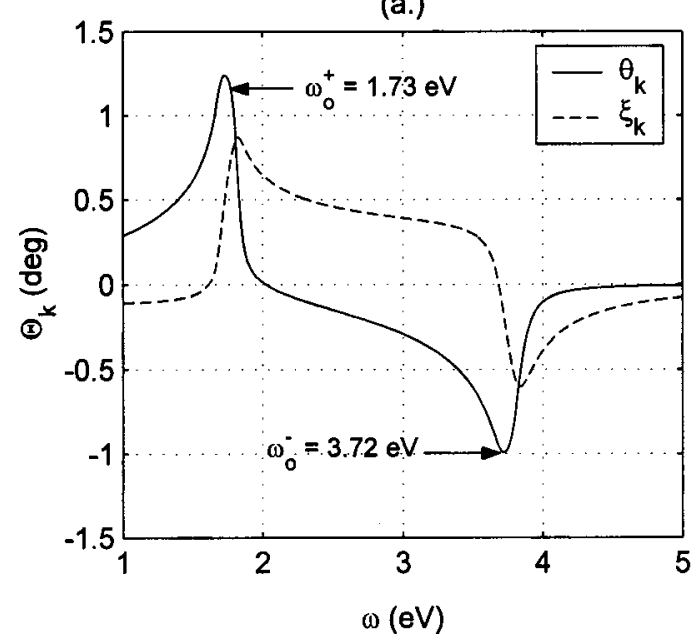

(c.)

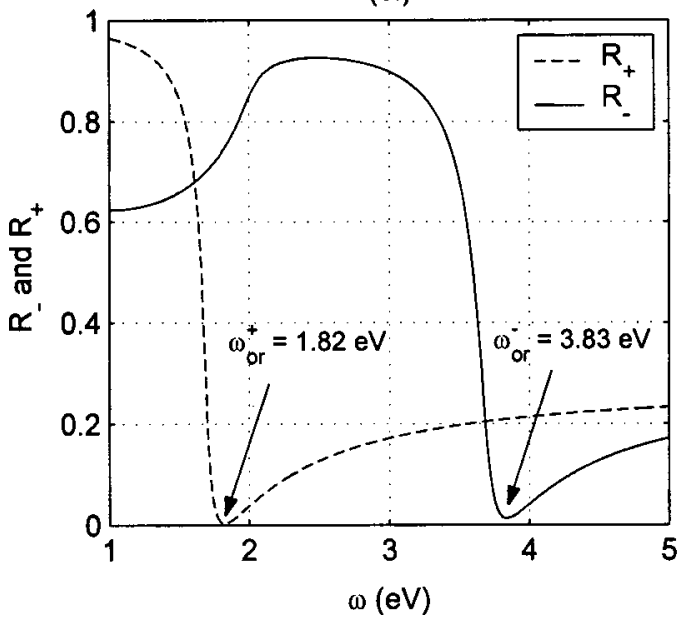

(e.)

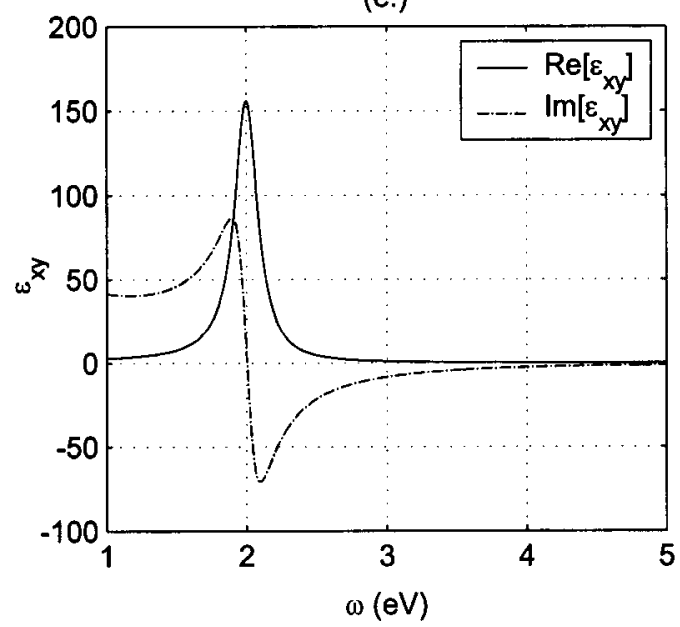

(b.)

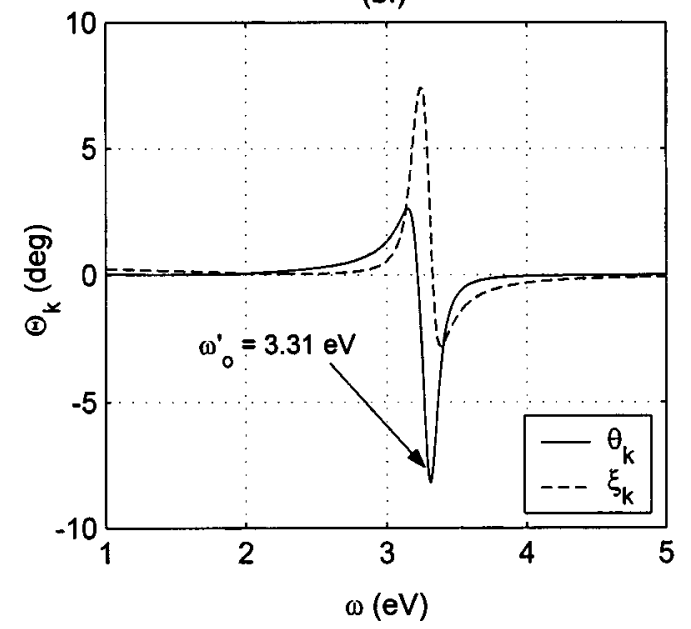

(d.)

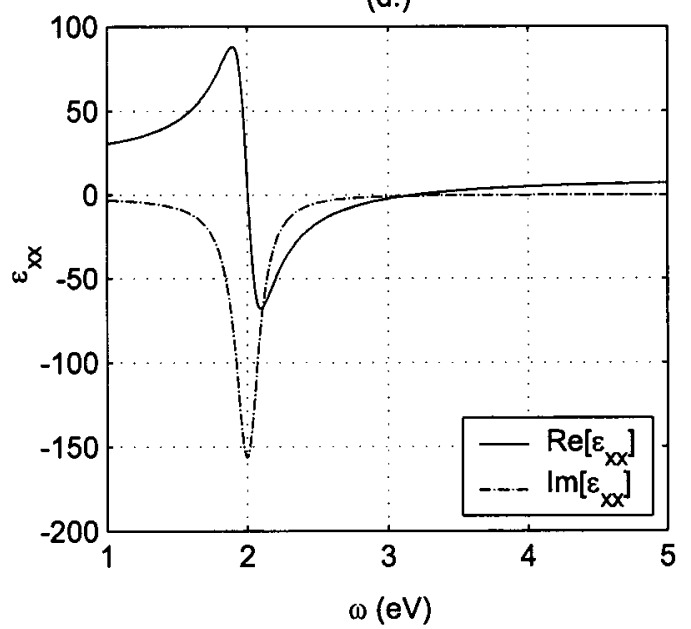

(f.)

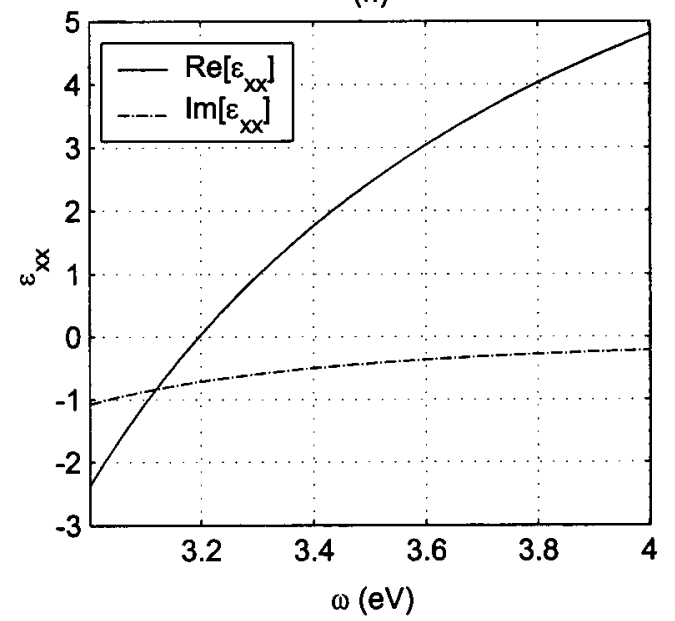

FIG. 4. (a) MOKE parameters using Eqs. (3) and (4). (b) MOKE parameters using Eq. (8). (c) Reflectivity. (d) Diagonal dielectric tensor component ( $\varepsilon_{x x}$ ). (e) Off-diagonal dielectric tensor component $\left(\varepsilon_{x y}\right)$. (f) Zoom in view of $\varepsilon_{x x}$; constants used: $\varepsilon_{\infty}=10, \omega_{p}=2.5 \mathrm{eV}, \omega_{c}=2 \mathrm{eV}$, and $\gamma=0.1 \mathrm{eV}$.

and predicted values is much greater. Also notice that in Fig. 3(a) for $\omega_{p} / \omega_{c}=5$, the $\omega_{o} / \omega_{c}$ curve is in closer proximity to the $\omega_{o r} / \omega_{c}$ curve than to either $\omega_{o 1} / \omega_{c}$ or $\omega_{o 2} / \omega_{c}$. The higher discrepancies seen in Fig. 3 between the numerically obtained $\omega_{o} / \omega_{c}$ and the predicted $\omega_{o 1} / \omega_{c}, \omega_{o 2} / \omega_{c}$ for decreasing $\omega_{p} / \omega_{c}$ can be explained based on Fig. 4.

In Fig. 4, we plot KR, KE, reflectivity, and dielectric tensor elements as a function of $\omega$, for $\varepsilon_{\infty}=10, \gamma=0.1 \mathrm{eV}$, $\omega_{c}=2 \mathrm{eV}$, and $\omega_{p}=2.5 \mathrm{eV}$. Note the higher value of the assigned cyclotron frequency as compared to the case illustrated in Fig. 1. The cyclotron resonance effect is seen in Figs. 4(d) and 4(e) as expected from Eqs. (10) and (2). As mentioned earlier, on the application of a magnetic field the plasma reflection edge splits into two and the separation be- 
tween the two reflectivity minima increases linearly with the field. ${ }^{20}$ Therefore, the large $\omega_{c}$ value is responsible for the large reflection edge split seen in Fig. 4(c). This effect is also seen in the MOKE spectra of Fig. 4(a), where the large reflection edge split causes the spectral elongation of the MOKE resonance. Here, we denote the two different optical frequencies at which the two KR peaks (with opposite signs) occur as $\omega_{o}^{+}$and $\omega_{o}^{-}$. Also note, that $\omega_{o}^{+}$and $\omega_{o}^{-}$are in the vicinity of the $R^{+}$and $R^{-}$reflectivity minima, respectively, and that $\omega_{o r}^{+}-\omega_{o r}^{-} \approx \omega_{o}^{+}-\omega_{o}^{-} \approx \omega_{c}$. Now, notice that the effect of the large reflection edge split on the MOKE spectrum is not at all seen in Fig. 4(b). Instead a MOKE enhancement effect is seen at $\omega_{o}^{\prime}=3.31 \mathrm{eV}$, where $\operatorname{Re}\left[\varepsilon_{x x}\right] \approx 1$. However in the case of Fig. 4(a) the optimum KR will occur at $\omega_{o}^{+}$ $=1.73$, and a second peak of slightly lesser magnitude and opposite sign occurs at $\omega_{o}^{-}=3.72 \mathrm{eV}$. This quite clearly implies that the approximation of Eq. (8) breaks down in the event of a large reflection edge split.

The reasons for the discrepancies seen between the MOKE spectra of Figs. 4(a) and 4(b) are as follows. First, note that Eq. (8) was used to obtain the FH condition. However, as the magnitude of $\omega_{c}$ approaches that of $\omega_{p}$, the FH condition for MOKE resonance gradually breaks down, as Eq. (8), which is an approximation of Eqs. (3) and (4), itself breaks down. This is attributed to the fact that while deriving Eq. (8) it was assumed that $\varepsilon_{x x} \gg \varepsilon_{x y} .{ }^{18}$ This assumption is no longer valid when the cyclotron resonance and plasma resonance are in close proximity of each other, as clearly seen in Figs. 4(d) and 4(e). Second, the approximation that $\operatorname{Im}\left[\varepsilon_{x x}\right] \approx 0$, is also no longer valid in the vicinity of a cyclotron resonance. The reason for this becomes apparent by taking a close look at the denominator of the imaginary term in Eq. (11).

In addition, the above-mentioned reasons can also qualitatively explain the increasing disagreement between the numerically obtained $\omega_{o} / \omega_{c}$, and the predicted $\omega_{o 1} / \omega_{c}$, $\omega_{o 2} / \omega_{c}$, with decreasing $\omega_{p} / \omega_{c}$, seen in Fig. 3. Also, for the computed MOKE spectra (for a single substrate) in Ref 23, FH's resonance condition was held at all incident photon energies by its appropriate coupling with the cyclotron frequency, yet the KR was seen to decrease with increasing incident photon energy. This phenomenon is also attributed to the corresponding decrease in $\omega_{p} / \omega_{c}$.

Thus, whenever $\omega_{c} \sim \omega_{p}$, large MOKE resonance no longer occurs at $\operatorname{Re}\left[\varepsilon_{x x}\right] \approx 1$. Therefore in the event of a large reflection edge split, instead of using the $\operatorname{Re}\left[\varepsilon_{x x}\right] \approx 1$ resonance condition, we propose the following alternative.

A more general approach can be adopted in predicting the occurrence of resonance like enhancements in the Kerr spectrum by reverting a step back from Eq. (8) to Eq. (7) and using the $\operatorname{Re}\left[\varepsilon_{+} \cdot \varepsilon_{-}\right]=1$ condition. This resonance condition follows from $\tilde{n}_{+} \cdot \tilde{n}_{-}=1$, which can be deduced by inspection from Eq. (7). An expression for the optical frequency at which the above mentioned MOKE resonance occurs is derived next by using the $\operatorname{Re}\left[\varepsilon_{+} \cdot \varepsilon_{-}\right]=1$ condition in Eq. (9)

$$
\begin{aligned}
& X \omega^{4}-(2 i X \gamma) \omega^{3}-\left(2 \omega_{p}^{2}+X \omega_{c}^{2}+X \gamma^{2}\right) \omega^{2}+\left(2 i \gamma \omega_{p}^{2}\right) \omega+\omega_{p}^{4} \\
& \quad=0,
\end{aligned}
$$

(a.)

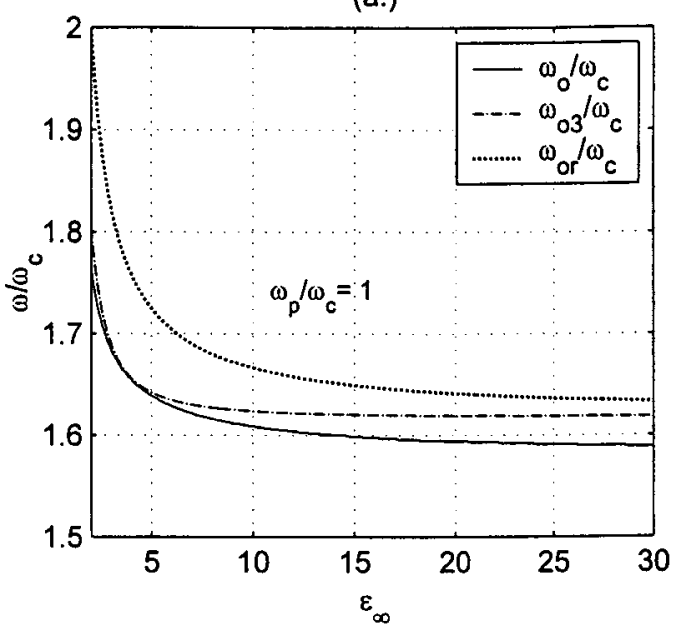

(b.)

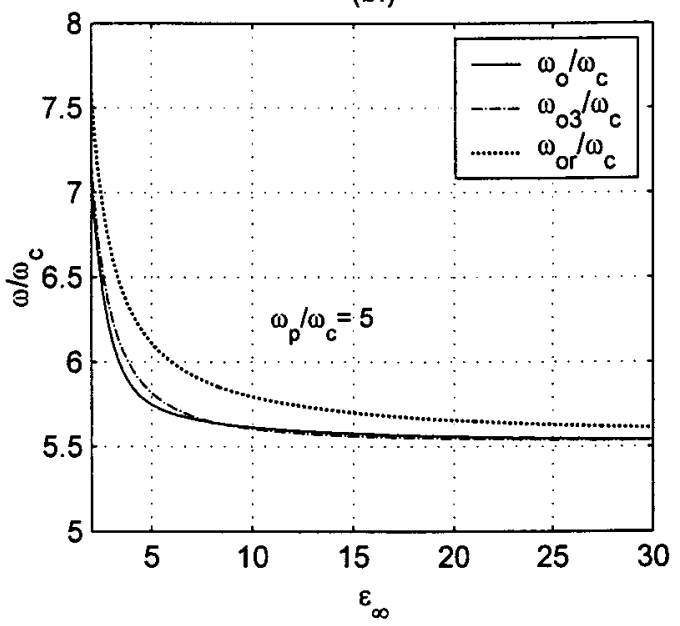

(c.)

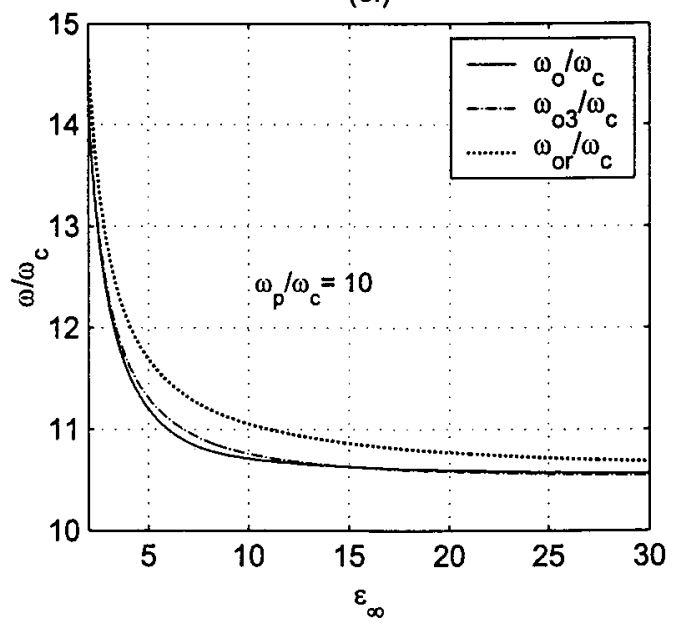

FIG. 5. Normalized comparison of the numerically obtained opticalfrequencies of KR maxima using Eq. (3), against Eqs. (13), (16), and (19) as a function of $\varepsilon_{\infty}$ for various $\omega_{p} / \omega_{c}$.

where $X=1-1 / \varepsilon_{\infty}^{2}$.

The damping constant $\gamma$ can be ignored, provided it is small. This enables us to bring Eq. (18) into a simpler quadratic form. By solving for $\omega$, we obtain 


$$
\begin{aligned}
\omega_{o 3}= & {\left[\frac{\omega_{c}^{2}}{2}+\frac{\omega_{p}^{2}}{X} \pm \frac{1}{2 X}\right.} \\
& \left.\times \sqrt{4 \omega_{p}^{4}(1-X)+X^{2} \omega_{c}^{4}\left(1+2 \omega_{p}^{2} / X \omega_{c}^{2}\right)}\right]^{1 / 2} .
\end{aligned}
$$

It is important to note that the use of - or + signs in Eq. (19) will subsequently yield either $\omega_{o}^{+}$or $\omega_{o}^{-}$[as indicated in Fig. 4(a)]. The validity of Eq. (19) with the + sign is tested next, over a range of optical constants, in Fig. 5. A comparison against the $\omega_{o r} / \omega_{c}$ curve has also been made. It is worth noting that the $\omega_{o 3} / \omega_{c}$ curve appears to be in good agreement with the $\omega_{o} / \omega_{c}$ curve, even for the low value of $\omega_{p} / \omega_{c}=1$. Therefore, we conclude that the $\operatorname{Re}\left[\varepsilon_{+} \cdot \varepsilon_{-}\right]=1$ condition is more general than the $\operatorname{Re}\left[\varepsilon_{x x}\right]=1$ condition, since much better comparative results are seen even for large reflection edge splitting effects (small $\left.\omega_{p} / \omega_{c}\right)$.

\section{SUMMARY}

In this article analytical expressions, in the framework of the Drude model, which predict the spectral occurrence of the Kerr rotation maxima, are obtained. These expressions, [Eq. (13) and Eq. (19)], are based on $\operatorname{Re}\left[\varepsilon_{x x}\right]=1$ and $\operatorname{Re}\left[\varepsilon_{+} \varepsilon_{-}\right]=1$ conditions, respectively. On comparing Eq. (13) (and hence $\operatorname{Re}\left[\varepsilon_{x x}\right]=1$ ) to numerically obtained optical frequencies at which the Kerr rotation peaks, it is seen that the reliability of Eq. (13) is best when $\omega_{p} \gg \omega_{c}$. However, once the magnitude of $\omega_{c}$ approaches that of $\omega_{p}$, the spectral occurrence of the MOKE maxima moves away from the vicinity of $\operatorname{Re}\left[\varepsilon_{x x}\right]=1$; this is attributed to the inability of Eq. (8) to take large reflection edge splits into account. Under these conditions, Eq. (19) is found to be of much greater reliability. Hence we conclude that $\operatorname{Re}\left[\varepsilon_{+} \varepsilon_{-}\right]=1$ is a more general condition, as compared to $\operatorname{Re}\left[\varepsilon_{x x}\right]=1$, in predicting the spectral occurrence of the MOKE maxima. However, for most cases $\omega_{p} \gg \omega_{c}$, therefore Eq. (13) should be adequate. Another important conclusion drawn from the numerical analysis is that the proximity of $\operatorname{Re}\left[\varepsilon_{x x}\right] \approx 1$ and the plasma edge is subject to the magnitude of the background dielectric constant $\varepsilon_{\infty}$, especially at lower values of $\varepsilon_{\infty}$ (or for magneto-optic materials with low refractive indices). This is based on the observation that the spectral location of the MOKE peak moves away exponentially from the vicinity of the plasma edge, with decreasing $\varepsilon_{\infty}$ (therefore $\mathrm{x}$ is no longer $\sim 1$ ). In closing, we wish to state that, provided optical constants are available a priori and that the Drude model can account for the MOKE behavior of that material, Eqs. (13) and (19) could be useful for the design and simulation multilayer magneto-optic structures and also for magnetooptic experiments.

${ }^{1}$ P. Drude, The Theory of Optics (Tuebrer, Leipzig, 1900); (Dover, New York, 1959).

${ }^{2}$ E. D. Palik, S. Teitler, B.W. Henvis, and R.F. Wallis, Proceedings of the International Conference on the Physics of Semiconductors (The Institute of Physics and the Physical Society, Exeter, 1962), p. 288.

${ }^{3}$ S. E. Schnatterly, Phys. Rev. 183, 664 (1969).

${ }^{4}$ O. G. Rutkin and P. P. Syrinikov, Phys. Solid State 35, 900 (1993).

${ }^{5}$ H. Brandle, J. Schones, and F. Hulliger, Helv. Phys. Acta 62, 199 (1989).

${ }^{6}$ W. Reim, O. E. Husser, J. Schones, E. Kaldis, and P. Wachter, J. Appl. Phys. 55, 2155 (1984).

${ }^{7}$ S. Wittekoek, T. J. A. Popma, J. M. Robertson, and P. F. Bongers, Phys. Rev. B 12, 2777 (1975).

${ }^{8}$ A. Delin, O. Eriksson, B. Johansson, S. Auluck, and J. M. Wills, Phys. Rev. B 60, 14105 (1999).

${ }^{9}$ H. S. Bennet and E. A. Stern, Phys. Rev. 137, A448 (1965).

${ }^{10}$ J. L. Erskine and B. R. Cooper, Phys. Rev. B 8, 1239 (1973).

${ }^{11}$ P. Oppeneer, T. Maurer, J. Sticht, and J. Kubler, J. Phys.: Condens. Matter 6, 285 (1994).

${ }^{12}$ T. Katayama, Y. Suzuki, H. Awano, Y. Nishihara, and N. Koshizuka, Phys. Rev. Lett. 60, 1426 (1988).

${ }^{13}$ W. Reim and D. Weller, Appl. Phys. Lett. 53, 2453 (1988).

${ }^{14}$ H. Fiel and C. Haas, Phys. Rev. Lett. 58, 65 (1988).

${ }^{15}$ J. Schones and W. Reim, Phys. Rev. Lett. 60, 1988 (1988).

${ }^{16}$ H. Feil and C. Haas, Phys. Rev. Lett. 60, 1989 (1988).

${ }^{17}$ K. Egashira and T. Yamada, J. Appl. Phys. 45, 3643 (1974).

${ }^{18}$ P. Argyres, Phys. Rev. 97, 334 (1955).

${ }^{19}$ W. Reim and J. Schones, Magneto Optical Spectroscopy, Ferromagnetic Materials, Vol. 5 (Elsevier, New York, 1990).

${ }^{20}$ S. J. Buchsbaum, Proceedings of the International Conference on the Physics of Semiconductors: Symposium on Plasma Effect in Solids (Dunod, Paris, 1964).

${ }^{21}$ J. G. Mavroides, in Optical Properties of Solids, edited by F. Abeles (North Holland, Amsterdam, 1972), Chap. 7.

${ }^{22}$ B. Lax and G. B. Wright, Phys. Rev. Lett. 4, 16 (1960).

${ }^{23}$ A. De and A. Puri, J. Appl. Phys. 91, 9777 (2002). 